\title{
Design and Optimization of Microstrip Patch Antenna Using Oval Patch and Parasitic Elements
}

\author{
Sandip Shivaji Patilı, Prof. Umesh B. Pagare2, \\ 1. Department of Electronics and Telecomm Engineering, Gangamai College of Engineering, Nagaon, Dhule, India. \\ 2. Department of Electronics and Telecomm Engineering, Gangamai College of Engineering, Nagaon, Dhule, India.
}

\begin{abstract}
We are use two microstrip patch antenna in order to examine the importance of parasitic elements having most interested topic. However, both antenna having same oval patch. While first has only oval patch and another has two additional oval parasitic elements. Then we check the angular rotation of the patch and the parasitic element. To arrange the angular rotation from $0^{0}$ to $60^{\circ}$ by increasing $15^{\circ}$ from this reading we check the return loss and radiation pattern result for each $15^{\circ}$ rotation of the angle. From a reading, we found that, the parasitic element down shift in -10dB impedance bandwidth and to increase in somewhat bandwidth. We found the highest bandwidth for the rotation angle of $15^{\circ}$ and $30^{\circ}$ respectively. From this maximum gain is $4.16 \mathrm{dbi}$. Therefore, we use both antennas for s-band radar application
\end{abstract}

Keywords: Microstrip patch, Oval patch, Angular rotation, Parasitic element, Parametric analysis.

\section{I) Introduction}

Microstrip patch antennas are used in various applications. Such that in radar, wireless communications, radio frequency identification (RFID) and also used in telemedicine because they are in small size, easy to fabricate, and low cost. [1]. Most of the work has been conducted to design and analyse different Shape. for a square microstrip patch antenna uses five different patch and shapes for wireless operations and in the Personal Communications Service (PCS), 3G, WiFi/WLAN/Bluetooth, and Long-term Evolution (LTE). Celik and Kurt [2] to design an integrated loop E-shaped microstrip patch antenna used for the Industrial Scientific Medical (ISM) band. in a range frequency between $2.200 \mathrm{GHz}$ to $2.500 \mathrm{GHz}$. for the energy harvesting applications. It is also suitable for the S, C, and X band applications. Feng et. al. [3] say that in microstrip patch antenna consisting of two hybrid shape radiation patches and a pair of capacitances loaded loop (CLL) resonators for 5.8 $\mathrm{GHz}$ WLAN operation. Liu et. al. [4] to perform a microstrip patch antenna with a radiating patch consisting of two rectangular parts connected to a ground plane. They achieve a bandwidth by adjusting the dimensions of the radiating patch. Wang et. al. [5] say to design a microstrip patch antenna with a long rectangular patch shorted to a ground plane. The antenna operates in TM03 and TM11 modes, and has a wide bandwidth along with a high gain. Antenna operates at $1.9 \mathrm{GHz}$ and it is circularly polarized. In addition, it manufactured through 3D printing technology.

More studies illustrate the usage of parasitic elements in microstrip patch antennas two identical parasitic metal strips are asymmetrically located along with two radiating edges of a rectangular microstrip patch antenna. Two coupling slots are also etched adjacent to the strips on the ground plane to realize energy transmission. Parasitic elements are used to improve the bandwidth of microstrip patch antennas and excite new resonant modes [6]. The structure resonates between 13.8-17.4 GHz. [7] analyse a disk patch antenna with two circular parasitic elements in single layer and multilayer configurations. [8] Propose a modified L-strip fed microstrip patch antenna for wideband applications. They incorporate dual U-shaped slots in the radiating patch along with two square parasitic elements on a Substrate, and conclude that the antenna with the parasitic elements has a wider $-10 \mathrm{~dB}$ impedance bandwidth than the configuration without the parasitic elements. In another study [9], a microstrip patch antenna with a rectangular patch and two smaller parasitic patches is investigated. .differential-fed microstrip patch antenna with complementary split ring resonators and a set of complementary loaded rectangular parasitic elements. Bandwidth of the antenna ranges from $5.3500 \mathrm{GHz}$ to $7.0000 \mathrm{GHz}$. Their analysis reveals that the multilayer configuration has a wider bandwidth and higher radiation levels than those of the single layer configuration. The antenna operates in TM30 and TM40 modes

By adjusting the dimensions of the parasitic patches and profile of the microstrip patch antenna, 
it is possible to control the frequency bandwidth. a microstrip patch antenna comprised of an inverse Sshape meander line connected with a rectangular box is designed for Internet of Things (IoT) applications at $2.4 \mathrm{GHz}$ ISM band. A rectangular parasitic patch, which has a high impact on the frequency bandwidth, is included in the design. explore a single layer, wideband, differential-fed microstrip patch antenna with complementary split ring resonators and a set of complementary loaded rectangular parasitic elements. Bandwidth of the antenna ranges from $5.3500 \mathrm{GHz}$ to $7.0000 \mathrm{GHz}$.

From this study, we propose two microstrip patch antennas with oval patches. One antenna also has two co-planar oval parasitic elements located on two sides of the patch. We first examine the effect of parasitic elements on the antennas, performance in terms of bandwidth. Next, we analyse the case when the patch and the parasitic elements are rotated in a defined range and increment. We obtained simulation results and Analysis the results. By obtaining the effect of rotation, it might be possible to determine the optimal location of the patch of a microstrip patch antenna during design processes. Other design parameters such as feed location should be taken into account simultaneously.

From this study is as following Section II describes the design process of the proposed antennas as well as the impact of parasitic elements. Section III explores the effect of angular rotation of the patch and the parasitic elements for one designed antenna. Section IV includes a discussion on the obtained simulation results. Finally, Section V concludes the paper.

\section{II) ANTENNAS AND IMPACT OF PARASITIC ELEMENTS}

We design two microstrip patch antennas in order to analyse the impact of parasitic elements. The first antenna, Shown in Figure 1, is a square antenna, and has an oval patch only. Width (and length) of the antenna is $34 \mathrm{~mm}$. Semi-major axis length and semi-minor axis length of the oval patch are $13.98 \mathrm{~mm}$ and $6 \mathrm{~mm}$, respectively. Substrate has a relative permittivity of 15.5 with a loss tangent of 0.0001 . Substrate height is 6.18 $\mathrm{mm}$. The antenna is fed by a 50 -ohm coaxial probe located at $(3.5,0.5) \mathrm{mm}$ in $\mathrm{x}-\mathrm{y}$ plane with respect to the centre of the oval patch denoted by $(0,0) \mathrm{mm}$. Circles on the patch of the antennas given in Figure 1 show the feed locations. The structure operates between 2.8850 $3.2750 \mathrm{GHz}$ with a fractional bandwidth (FBW) of $12.66 \%$. Fractional bandwidth is given in Equation 1

$$
\mathrm{FBW}=\frac{2\left(f_{\mathrm{H}}-f_{\mathrm{L}}\right)}{\left(\mathrm{f}_{\mathrm{H}}+\underline{\mathrm{f}}_{\mathrm{L}}\right)}
$$

Therefor $f_{H}$ is the upper frequency end and $f_{L}$ are the lower frequency ends of $-10 \mathrm{~dB}$ impedance bandwidth, respectively.

The second antenna in Figure 1 has two coplanar oval parasitic elements located on two sides of the oval patch. These parasitic elements are located at $(-12,0)$ $\mathrm{mm}$ and $(12,0) \mathrm{mm}$ in $\mathrm{x}-\mathrm{y}$ plane. Their semi-major axis lengths and semi-minor axis lengths are $8 \mathrm{~mm}$ and $2 \mathrm{~mm}$, respectively. The structure operates between $2.8687-3.2733 \mathrm{GHz}$ with a FBW of $13.17 \%$. All remaining properties of the second antenna are the same as those of the first antenna. We evaluate the impact of parasitic elements in Section IV.

\section{III) PARAMETRIC ANALYSIS FOR ANTENNA WITH PARASITIC ELEMENTS}

In this section, we carry out a parametric analysis for the antenna with the parasitic elements in order to investigate the impact of angular rotation on the antenna performance. In particular, we rotate the antenna patch and the parasitic elements in $15^{\circ}$ increments from $0^{\circ}$ to $60^{\circ}$ clockwise direction, and check the simulation results. For all simulations, the feed location is $(3.5,0.5) \mathrm{mm}$ in $\mathrm{x}-\mathrm{y}$ plane. We utilize the topology shown in the bottom figure of Figure 1. In that figure, we presume the positive y axis points to $0^{\circ}$. The antenna resonates between $2.8687-$ $3.2733 \mathrm{GHz}$

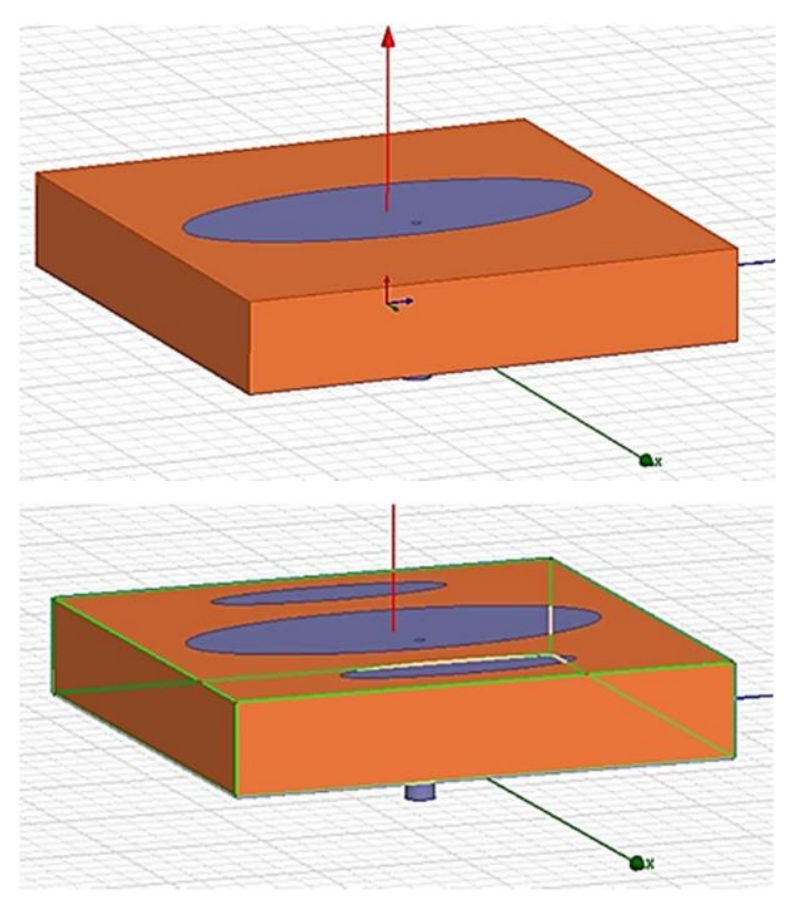


Figure 1. Designed microstrip patch antennas Without parasitic elements (top), and with parasitic elements (bottom).with a FBW of $13.17 \%$, as shown in Section II.

Figure 2 demonstrates the return loss versus frequency. And

Figure 3 shown the radiation patterns at the resonant frequency of $3.2000 \mathrm{GHz}$ for $\phi=0^{\circ}$ and $\phi=90^{\circ}$ planes.

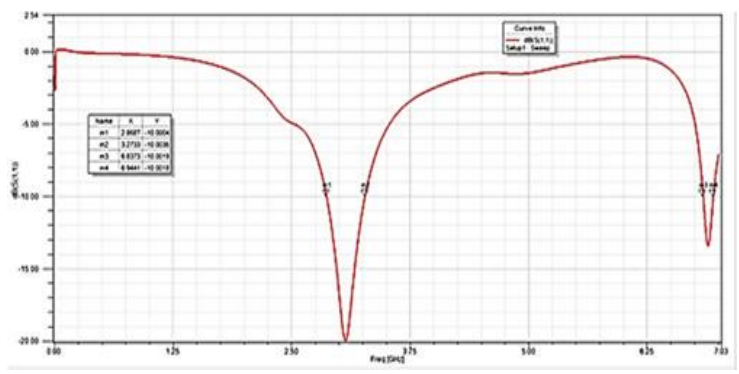

Figure 2. Return loss versus frequency for antenna with parasitic elements (rotation angle: $0^{\circ}$ ).

There fore we conclude that antenna with the patch and the parasitic elements rotated by $15^{\circ}$ clockwise direction. In this case, the antenna operates between $2.8742-3.2811 \mathrm{GHz}$ with a FBW of $13.22 \%$.

Figure 4 shows the return loss versus frequency.

Figure 5 shows the radiation patterns at the resonant frequency of $3.1800 \mathrm{GHz}$ for $\phi=0^{\circ}$ and $\phi=90^{\circ}$ planes.

After, we check the antenna with the patch and the parasitic elements rotated by $30^{\circ}$ clockwise direction. The antenna operates between $2.8551-3.2125 \mathrm{GHz}$ with a FBW of $11.78 \%$.

Figure 6 shown the return loss versus frequency. And Figure 7 shown the radiation patterns at the resonant frequency of $3.0600 \mathrm{GHz}$ for $\phi=0^{\circ}$ and $\phi=90^{\circ}$ planes.

We check the antenna with the patch and the parasitic elements rotated by $45^{\circ}$ clockwise direction. In that case, the antenna operates between 2.8067-3.0994 $\mathrm{GHz}$ with a FBW of $9.91 \%$.

Figure 8 shown the return loss versus frequency.

Figure 9 shown the radiation patterns at the resonant frequency of $2.9400 \mathrm{GHz}$ for $\phi=0^{\circ}$ and $\phi=90^{\circ}$ planes

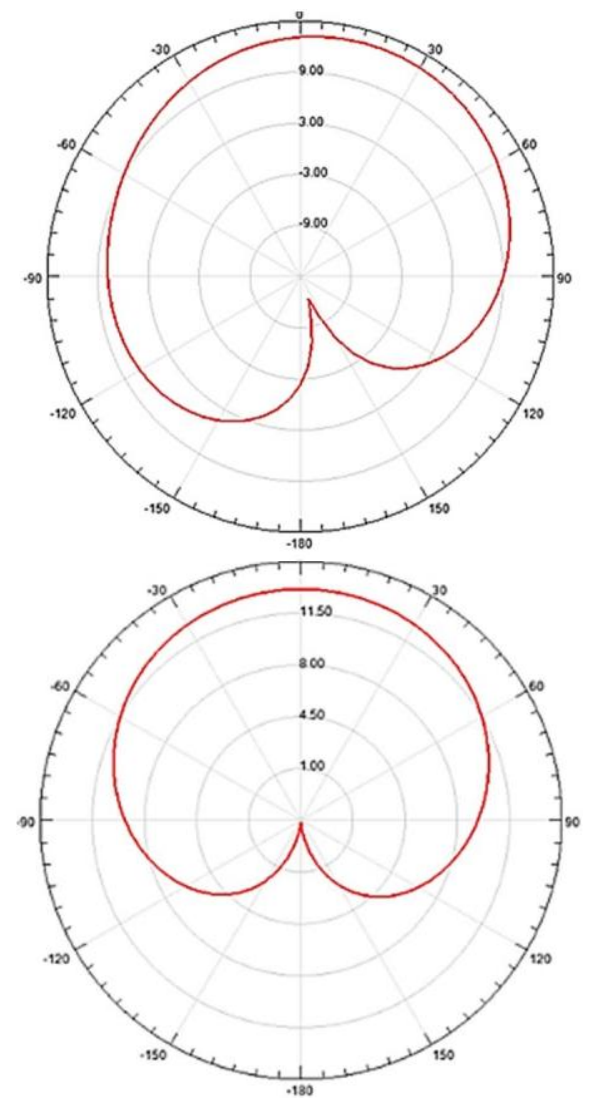

Figure 3. Radiation patterns for $\phi=0^{\circ}$ (top) and $\phi=90^{\circ}$ (bottom) planes considered for antenna with parasitic elements (rotation angle: $0^{\circ}$ ).

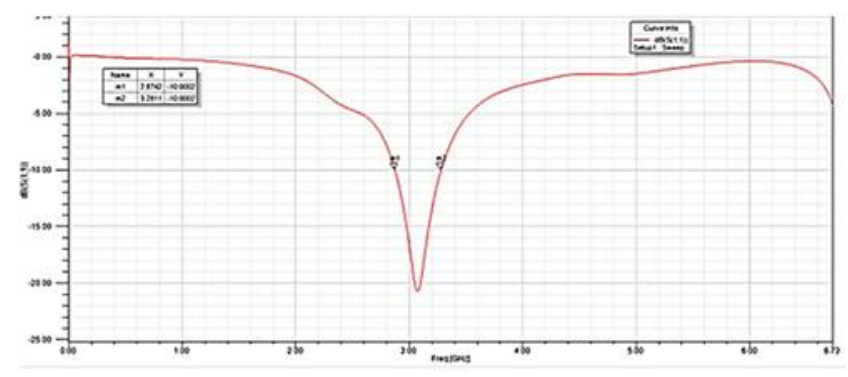

Figure 4. Return loss versus frequency for antenna with parasitic elements (rotation angle: $15^{\circ}$ ).

Last, we explore the antenna with the patch and the parasitic elements rotated by $60^{\circ}$ clockwise direction. The antenna operates between $2.7693-2.9890 \mathrm{GHz}$ with a FBW of $7.63 \%$.

Figure 10 shows the return loss versus frequency.

Figure 11 shown the radiation patterns at the resonant frequency of $2.8800 \mathrm{GHz}$ for $\phi=0^{\circ}$ and $\phi=90^{\circ}$ planes.

Figure 12 shown the resonant frequency shift for the rotation angles of $0^{\circ}, 15^{\circ}, 30^{\circ}, 45^{\circ}$ and $60^{\circ}$. We include all evaluation results in Section IV. 


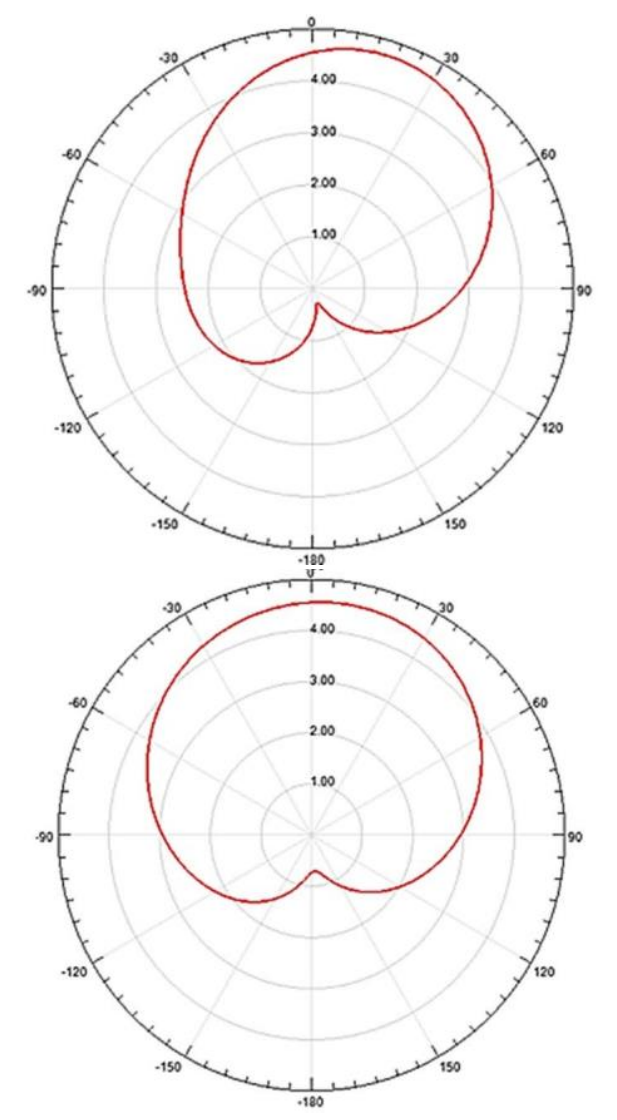

Figure 5. Radiation patterns for $\phi=0^{\circ}$ (top) and $\phi=90^{\circ}$ (bottom) planes considered for antenna with parasitic elements (rotation angle: $15^{\circ}$ ).

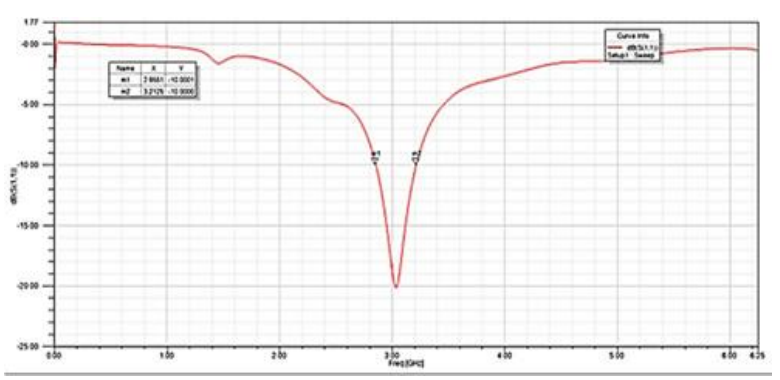

Figure 6. Return loss versus frequency for antenna with parasitic elements (rotation angle: $30^{\circ}$ ).

\section{IV) DISCUSSION}

We designed microstrip patch antennas with and without the parasitic elements in Section II. We conclude that the operation range of the antenna without the parasitic $(2.8850-3.2750 \mathrm{GHz})$ is shifted down to $2.8687-3.2733 \mathrm{GHz}$ when the parasitic elements are included. The reason for this shift is the increase in the antenna capacitance when the parasitic elements are added to the antenna. Therefore,
Equation 2 gives the resonant frequency of an antenna

\section{(2)}

$$
\text { Fr = 1/2Pi Root La Ca }
$$

where $f_{r}$ is the resonant frequency of the antenna, $L a$ is the antenna inductance, and $C a$ is the antenna capacitance. Therefore, an increase in the antenna capacitance results in a decrease in the resonant frequency. Also, conclude that the parasitic elements enhances the FBW. The FBW for the antennas with and without the parasitic elements are $13.17 \%$ and $12.66 \%$, respectively.
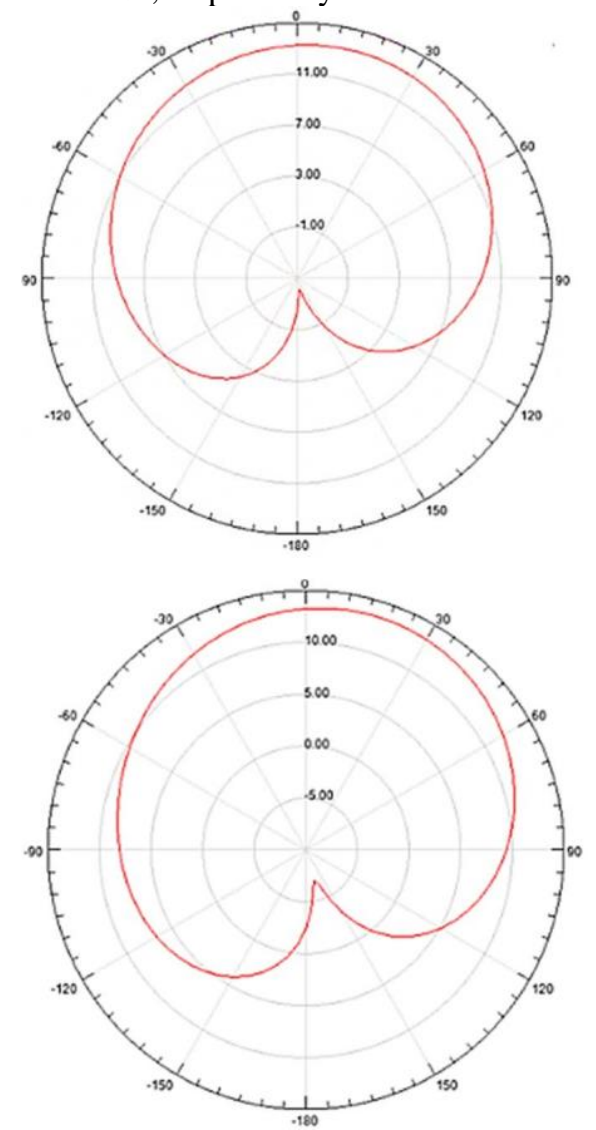

Figure 7. Radiation patterns for $\phi=0^{\circ}$ (top) and $\phi=90^{\circ}$ (bottom) planes considered for antenna with parasitic elements (rotation angle: $30^{\circ}$ ).

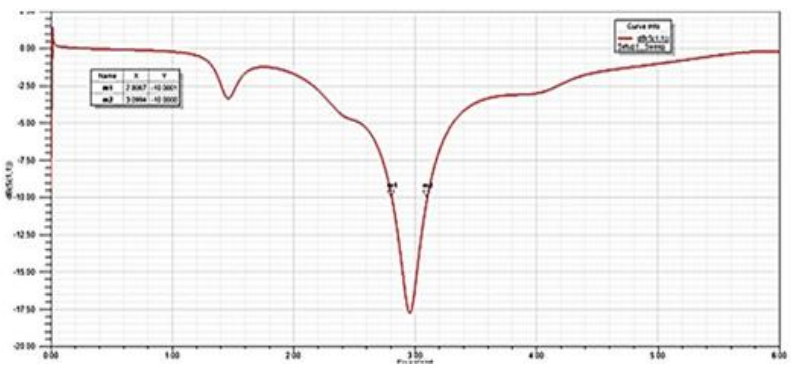


Figure 8. Return loss versus frequency for antenna with parasitic elements (rotation angle: $45^{\circ}$ ).

We observe that semi-hemispherical coverage for both $\phi=0^{\circ}$ and $\phi=90^{\circ}$ planes in all radiation patterns in Section III. The Comparison of radiation patterns in Figure 3 and Figure 5. radiation level decrease for the rotation angle of $15^{\circ}$. The reason for due to the stationary feed location (in both cases the coaxial feed is located at $(3.5,0.5) \mathrm{mm})$, which could result in a impedance mismatch. Also, the radiation pattern for $\phi=0^{\circ}$ plane in Figure 5 is more than that for

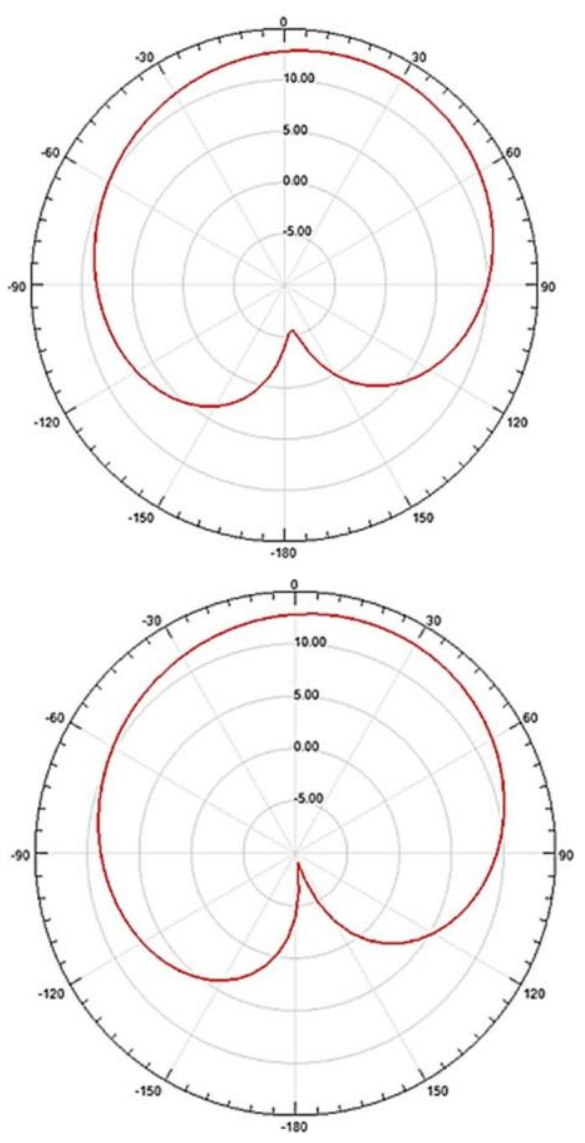

Figure 9. Radiation patterns for $\phi=0^{\circ}$ (top) and $\phi=90^{\circ}$ (bottom) planes considered for antenna with parasitic elements (rotation angle: $45^{\circ}$ ).

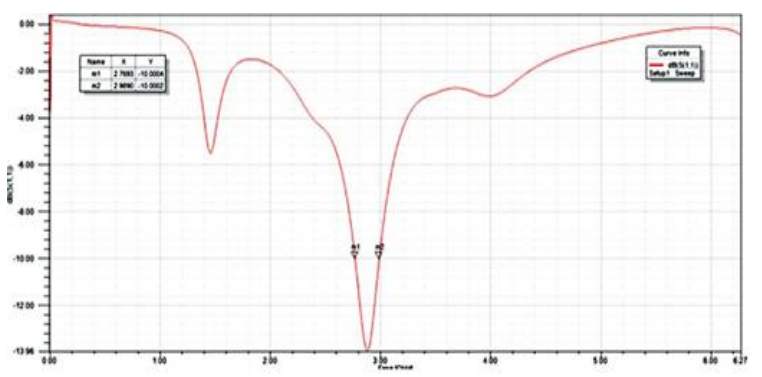

Figure 10. Return loss versus frequency for antenna with parasitic elements (rotation angle: $60^{\circ}$ ).

Figure 3. Radiation patterns and the radiation levels for the rotation angle of $30^{\circ}$ (Figure 7) are similar to those for the rotation angle of $0^{\mathrm{O}}$ (Figure 3), which means a proper impedance matching. Maximum radiation directions of the radiation patterns for the rotation angle of $45^{\circ}$ (Figure 9) are slightly different from those for the rotation angle of $30^{\circ}$ (Figure 7). In Figure 9, maximum radiation occurs at $20^{\circ}$ for $\phi=0^{\circ}$ plane while it occurs at $15^{\circ}$ for the same plane in Figure 7. Similarly, maximum radiation occurs at $10^{\circ}$ for $\phi=90^{\circ}$ plane in Figure 9 while it occurs at $15^{\circ}$ for the same plane in Figure 7. Radiation patterns for the rotation angle of $60^{\circ}$ in Figure 11 are also Maximum radiation level occurs at $30^{\circ}$ for $\phi=0^{\circ}$ plane, and it occurs at $25^{\circ}$ for $\phi=90^{\circ}$ plane. More, radiation levels in Figure 11 are significantly lower than those in the previous radiation pattern figures, which indicates a impedance mismatch.
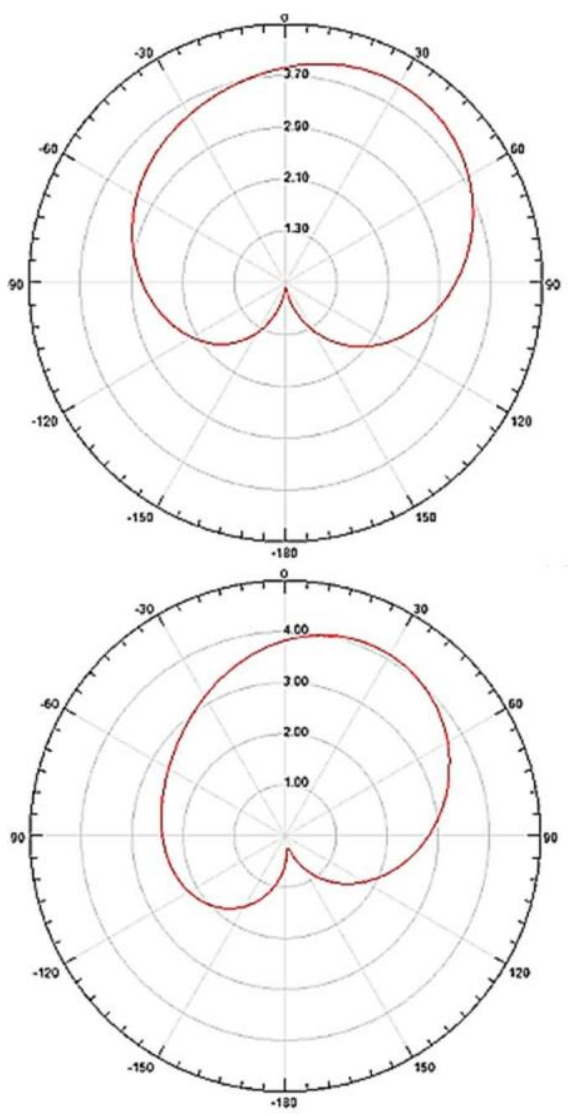

Figure 11. Radiation patterns for $\phi=0^{\circ}$ (top) and $\phi=90^{\circ}$ (bottom) planes considered for antenna with parasitic elements (rotation angle: $60^{\circ}$ ). 


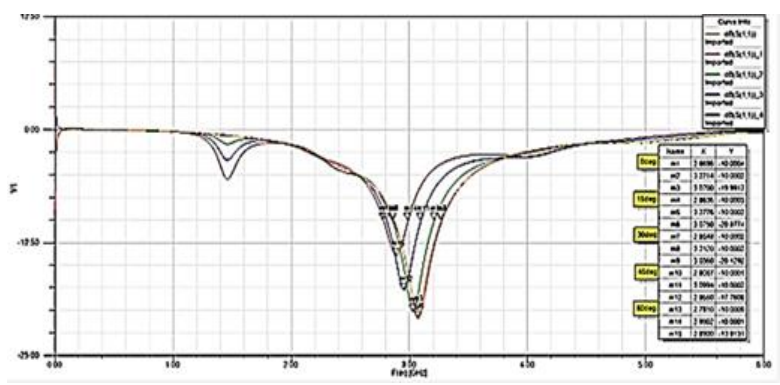

Figure 12. Resonant frequency variation for rotation angles of $0^{\circ}, 15^{\circ}, 30^{\circ}, 45^{\circ}$ and $60^{\circ}$.

From all angle rotation, the highest radiation levels and maximum gain is to be $4.16 \mathrm{dBi}$ for the rotation angle of $30^{\circ}$.

We obtain the highest FBW as $13.22 \%$ for the rotation angle of $15^{\circ}$. Figure 12 show a gradual decrease in the resonant frequency as the rotation angle increases. due to an increase in the antenna current path, which results in an antenna inductance increase while a decrease in the resonant frequency with increasing rotation angles (from Equation 2). Since antenna capacitance does not change with rotation angles, only increasing $L_{a}$. Should be a resonant frequency decrease with increasing rotation angles.

From Figure 12, we also conclude that another resonance formation at $1.4700 \mathrm{GHz}$ for the rotation angles of $30^{\circ}, 45^{\circ}$ and $60^{\circ}$. Return

loss level of that resonance formation reaches to -5.6 $\mathrm{dB}$ for the rotation angle of $60^{\circ}$.

\section{V) CONCLUSION}

We design two microstrip patch antennas with and without parasitic elements. Both antennas have the same oval patch. The parasitic elements are also oval.as per design properties along with the $-10 \mathrm{~dB}$ impedance bandwidth and the FBW for both antennas. after that, we carry out a parametric analysis for the antenna with parasitic elements in order to better understand the impact of angular rotation of the patch and the parasitic elements. Rotation angles are $0^{\circ}, 15^{\circ}$, $30^{\circ}, 45^{\circ}$ and $60^{\circ}$. We conclude the Return loss and the radiation pattern results for each rotation angle. Then we finalised the overall simulation results. Both antennas (with and without the parasitic elements) could be used for S-band radar applications.

\section{REFERENCES}

[1] C. A. Balanis, "Antenna theory: analysis and design," Wiley, New York, USA, 2012.

[2] K. Celik and E. Kurt, "A novel meander line integrated E-shaped for energy harvesting applications," International Journal of RF and Microwave Computer-Aided Engineering, pp. 1-10, 2018.

[3] S. Feng, L. Zhang, Z. B. Weng, and Y. C. Jiao, "A compact broadband differential-fed microstrip patch antenna with $5.8 \mathrm{GHz}$ WLAN band- notched under quad-mode resonance," Microw. Opt. Technol. Lett., pp. 1-8, 2019.

[4] N. W. Liu, S. Gao, L. Zhu, L. Y. Ji, et. al., "Lowprofile microstrip patch antenna with simultaneous enhanced bandwidth, beam width, and crosspolarisation under dual resonance," IET Microwaves, Antennas \& Propagation, vol. 14, pp. 360-365, 2020.

[5] Z. Wang, J. Liu, and Y. Long, "A simple widebandwidth and high- gain microstrip patch antenna with both sides shorted," IEEE Antennas and Wireless Propagation Letters, vol. 18, pp. 1144-1148, 2019.

[6] L. Li, Y. Zhang, J. Wang, W. Zhao, et. al.,"Bandwidth and gain enhancement of patch antenna with stacked parasitic strips based on LTCC technology," International Journal of Antennas and Propagation, vol. 2014, pp. 1-5, 2014.

[7] J. A. Ansari, N. P. Yadav, and P. Singh, "Analysis of disk patch antenna with parasitic elements in single and multilayer structures," Microw. Opt. Technol. Lett., pp. 865-870, 2010.

[8] H. W. Yu, Y. C. Jiao, D. Y. Li, and Z. B. Weng, "A $\mathrm{TM}_{30} / \mathrm{TM} 40$-mode pattern-reconfigurable microstrip patch antenna for wide beam coverage," IEEE Transactions on Antennas and Propagation, vol. 67, no. 11, pp. 7121-7126, 2019.

[9] M. S. Islam, M. T. Islam, MD. A. Ullah, G.K. Beng, et. al.,"A modified meander line microstrip patch antenna with enhanced bandwidth for 2.4 GHz ISM-band internet of things (IoT) applications," IEEE Access, vol. 7, pp. 127850-127861, 2019. 